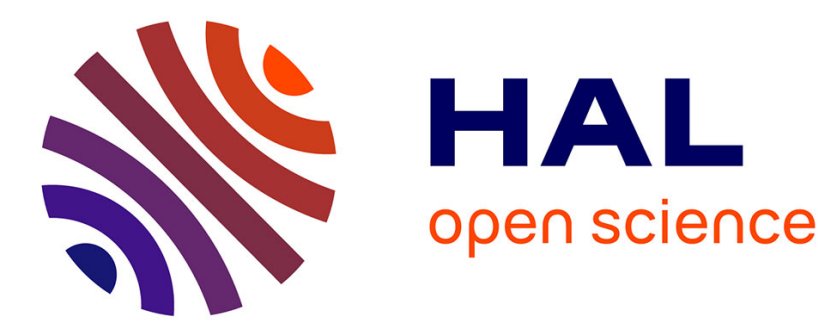

\title{
Polarization control in spun and telecommunication optical fibers
}

\author{
E. Assemat, D. Dargent, Antonio Picozzi, Hans-Rudolf Jauslin, Dominique \\ Sugny
}

\section{To cite this version:}

E. Assemat, D. Dargent, Antonio Picozzi, Hans-Rudolf Jauslin, Dominique Sugny. Polarization control in spun and telecommunication optical fibers. Optics Letters, 2011, 36, pp.4038. hal-00699910

\section{HAL Id: hal-00699910 https://hal.science/hal-00699910}

Submitted on 21 May 2012

HAL is a multi-disciplinary open access archive for the deposit and dissemination of scientific research documents, whether they are published or not. The documents may come from teaching and research institutions in France or abroad, or from public or private research centers.
L'archive ouverte pluridisciplinaire HAL, est destinée au dépôt et à la diffusion de documents scientifiques de niveau recherche, publiés ou non, émanant des établissements d'enseignement et de recherche français ou étrangers, des laboratoires publics ou privés. 


\title{
Polarization control in spun and telecommunication optical fibers
}

\author{
Elie Assémat, Damien Dargent, Antonio Picozzi, Hans-Rudolf Jauslin, and Dominique Sugny* \\ Laboratoire Interdisciplinaire Carnot de Bourgogne (ICB), UMR 5209 CNRS-Université de Bourgogne, Dijon, France \\ *Corresponding author: dominique.sugny@u-bourgogne.fr \\ Received June 22, 2011; revised September 6, 2011; accepted September 15, 2011; \\ posted September 15, 2011 (Doc. ID 149754); published October 7, 2011

\begin{abstract}
We consider the counterpropagating interaction of a signal and a pump beam in a spun fiber and in a randomly birefringent fiber, the latter being relevant to optical telecommunication systems. On the basis of a geometrical analysis of the Hamiltonian singularities of the system, we provide a complete understanding of the phenomenon of polarization attraction in these two systems, which allows to achieve a control of the polarization state of the signal beam by adjusting the polarization of the pump. In spun fibers, all polarization states of the signal beam are attracted toward a specific line of polarization states on the Poincaré sphere, whose characteristics are determined by the polarization state of the injected backward pump. In randomly birefringent telecommunication fibers, we show that an unpolarized signal beam can be repolarized into any particular polarization state, without loss of energy. (c) 2011 Optical Society of America
\end{abstract} \\ OCIS codes: $\quad 190.4370,060.4370,190.0190$.
}

Conventional polarizers are known to achieve light polarization by wasting $50 \%$ of the energy of the beam. More recently, by exploiting the nonlinearity of certain materials, "universal polarizers" performing polarization of unpolarized light with 100\% efficiency have been proposed [1-3]. This phenomenon may be called "polarization attraction," in the sense that all input polarization configurations are transformed into a well-defined polarization state, without any loss of energy of the beam. Polarization attraction has been demonstrated, in particular, in an optical fiber system pumped at both ends by two counterpropagating beams [4]. Considering an isotropic optical fiber, it was shown that, for a given state of polarization (SOP) of the backward pump, all polarization states of the forward signal beam can be attracted toward a particular polarization state, which is determined by the SOP of the pump $[2, \underline{5}, \underline{6}]$. Recent studies revealed that an efficient polarization attraction may also occur in different types of optical fibers, like, e.g., highly birefringent spun fibers (HBSFs) [7] and in randomly birefringent fibers (RBFs) [요, $\underline{9}$.

From a different perspective, we recently showed that the phenomenon of polarization attraction can be explained through the analysis of the singularities of the stationary Hamiltonian dynamics of the system $[\underline{6}, \underline{10}, \underline{11}]$. This theoretical approach revealed that the stationary solutions of interest belong to a particular two-dimensional object of the corresponding phase space representation, the so-called singular torus, whose singular topology is at the origin of the process of attraction. These recently developed mathematical techniques $[\underline{12}, 13]$ proved remarkably efficient for the understanding of the phenomenon of polarization attraction in isotropic fibers $[6,10,11]$.

Our aim in this Letter is to provide a generalized comprehension of the phenomenon of polarization attraction by analyzing the concrete examples of HBSFs and RBFs recently considered in Refs. [7-9]. Making use of the mathematical techniques exploite $\bar{d}$ in $[6,10,11]$, we show that HBSFs exhibit a novel phenomenon of polarization attraction. As opposed to the conventional attraction processes discussed so far, in HBSFs, all SOPs of the signal beam are not attracted to a single point but toward $a$ specific line of polarization states of the Poincaré sphere. In the particular case of a vanishing ellipticity of the spun fiber, the stationary states of HBSFs become of the same type as the one of RBFs. The theory reveals in this case that an unpolarized signal beam can be polarized into any desired polarization state, which is determined by the injected SOP of the backward pump. Besides its fundamental interest, polarization attraction in RBFs is of great importance in optical telecommunication systems in order to achieve a repolarization of optical transmission lines without loss of energy [ㅇ, 9$]$.

The evolution of the counterpropagating waves in HBSFs and RBFs is ruled by [7]

$$
\left\{\begin{array}{l}
\frac{\partial \vec{S}}{\partial t}+\frac{\partial \vec{S}}{\partial z}=\vec{S} \wedge(\mathcal{I} \vec{S})+\vec{S} \wedge(\mathcal{J} \vec{J}) \\
\frac{\partial \vec{J}}{\partial t}-\frac{\partial \vec{J}}{\partial z}=\vec{J} \wedge(\mathcal{I} \vec{J})+\vec{J} \wedge(\mathcal{J} \vec{S})
\end{array},\right.
$$

where $\vec{S}$ and $\vec{J}$ are the Stokes vectors for the forward and backward waves, respectively, and $\wedge$ denotes the vector product. The radii of the forward and backward spheres, $S_{0}$ and $J_{0}$, which correspond to the signal and pump powers, are assumed to be identical in the following $\left(S_{0}=J_{0}\right)$.

Polarization attraction in HBSFs. In this case, the diagonal matrices $\mathcal{I}$ and $\mathcal{J}$ are given by $\mathcal{I}=$ Diag $\left(0,0,2 \sin ^{2} \phi-\cos ^{2} \phi\right)$ and $\mathcal{J}=\cos ^{2} \phi \times \operatorname{Diag}(1,-1,-2)$, where $\phi$ is the ellipticity of the fiber [7]. Because of the counterpropagating nature of the interaction, stationary boundary conditions $\vec{S}(z=0)$ and $\vec{J}(z=L)$ are imposed at the two ends of the fiber of length $L$. We normalized the problem with respect to the nonlinear interaction time $\tau_{0}=1 /\left(\gamma S_{0}\right)$ and length $\Lambda_{0}=v \tau_{0}$, where $\gamma$ is the nonlinear Kerr coefficient and $v$ the group velocity of the waves.

As in the case of conventional isotropic fibers $[\underline{6}, \underline{10}, \underline{11}]$, numerical simulations of Eq. (1) reveal that, irrespective of the initial conditions, the spatiotemporal dynamics exhibit a relaxation toward a stationary state. Our approach is based on the study of the singularities of the stationary Hamiltonian trajectories. In particular, we showed in Refs. $[\underline{6}, \underline{10}, \underline{11}]$ that the stationary states selected by the 
space-time dynamics lie on the surface of a singular torus, which can be viewed as a two-dimensional extension of the concept of separatrix, well known for systems with 1 degree of freedom. The singular torus thus plays the role of an attractor for the wave system $[\underline{6}, \underline{10}, \underline{11}]$. We refer the reader to Ref. [14] for a detailed presentation of the singular reduction theory applied to spun and telecommunication optical fibers. Here, this approach is only briefly explained. The Hamiltonian structure of the stationary Eq. (1) is defined by

$$
\begin{aligned}
H= & \cos ^{2} \phi\left(S_{2} J_{2}-S_{1} J_{1}+2 S_{3} J_{3}\right) \\
& -\frac{2 \sin ^{2} \phi-\cos ^{2} \phi}{2}\left(S_{3}^{2}+J_{3}^{2}\right) .
\end{aligned}
$$

The stationary system admits another constant of motion $K=S_{3}+J_{3}$, which makes the system Liouvilleintegrable $[12,13]$. The corresponding energy-momentum diagram for the HBSF is reported in Fig. 1(a) for $\phi=\pi / 4$. Besides the usual regular tori, the diagram $(H, K)$ reveals the presence of a line of singular bitori for $\phi \in] 0, \pi / 2[$. A bitorus is the union of two tori glued along a circle (see Fig. 1). The equation of the line of singular bitori in the diagram $(H, K)$ is

$$
H=\cos ^{2} \phi\left(K^{2}-1\right)-\frac{\sin ^{2} \phi}{2} K^{2} .
$$

This continuous line of singularities reveals the existence of a new phenomenon of polarization attraction. Indeed, for an isotropic optical fiber the diagram was characterized by an isolated singular torus, which in turn was shown to lead to a polarization attraction toward a unique (or a discrete set of) polarization state(s) of the signal $[10,11]$. Here, the presence of the line of singularities will be shown to lead to a polarization attraction toward a continuous line of polarization states on the
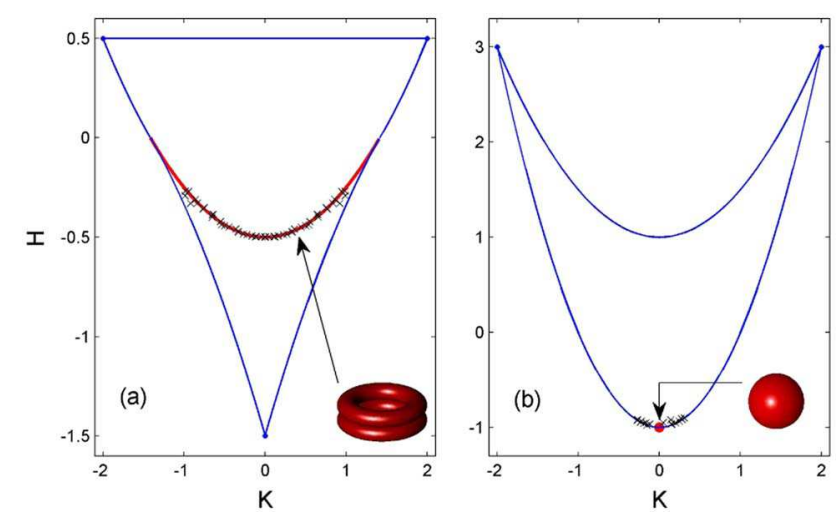

Fig. 1. (Color online) Energy momentum diagram $(H, K)$ for the HBSF with (a) $\phi=\pi / 4$ and (b) $\phi=0$. The (a) singular (red) line and the (b) singular (red) point, play the role of attractors for the space-time dynamics (1). The dark crosses locate the positions of the stationary states obtained by solving numerically the space-time Eq. (1) for different initial conditions of the signal $(L=5, \vec{J}(L)=(0,1,0))$. Note that, according to Eq. (4), the positions of the crosses satisfy $|K| \leq 1$. Each point of the singular red line in (a) is associated to a bitorus, while the singular red point at $K=0$ in (b) corresponds to a sphere of singular points (see the text).

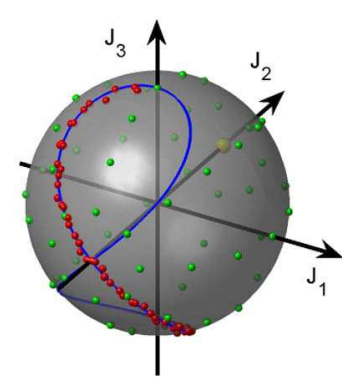

(a)

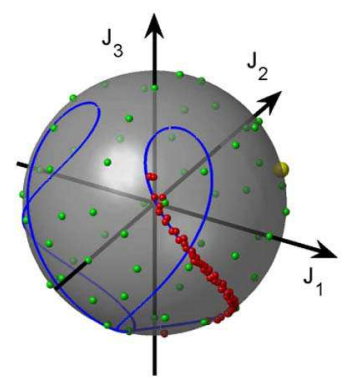

(b)
Fig. 2. (Color online) Polarization attraction toward a continuous line of polarization states for the HSBF: Numerical simulations of the spatiotemporal Eq. (1) on the Poincaré sphere [ $\phi=\pi / 4$ for (a) and $\phi=\pi / 5$ for (b) $]$. The green and red dots denote respectively the initial $(\vec{S}(0))$ and final $(\vec{S}(L))$ SOPs of the signal. The yellow dot denotes the fixed pump SOP: (a) $\vec{J}(L)=(0,1,0)$, (b) $\vec{J}(L)=(0.7,0.7,0)$ for $L=5$. The blue line is calculated analytically from our method.

Poincaré sphere. As explained below, some restrictions have to be added to this effect, which also depends on the value of $J_{3}$. We investigated this attraction by performing numerical simulations of the space-time Eq. (1). We considered 64 different initial SOPs of the signal $\vec{S}(z=0)$ uniformly distributed over the surface of the Poincaré sphere (green points in Fig. 2), while the SOP of the pump was kept fixed (yellow point). As illustrated in Fig. 2, all individual signal SOPs are attracted, at $z=L$, toward a specific continuous line on the surface of the Poincaré sphere (red points).

The line of polarization attraction can be calculated analytically from our approach. For a given pump SOP $\vec{J}(z=L)$, and using $S_{1}(L)^{2}+S_{2}(L)^{2}+S_{3}(L)^{2}=1$ and $K=S_{3}(L)+J_{3}(L)$, we can express the Hamiltonian (2) in terms of $K$ and of $S_{1}(L)$ [or alternatively $S_{2}(L)$ ]. Then, the variable $H$ can be eliminated by using Eq. (3), which thus gives a quadratic equation for the unknown $S_{1}(L)$ [or $\left.S_{2}(L)\right]$, whose coefficients depend on $K, \phi$, and $\vec{J}(z=L)$. This system can be solved analytically, which provides the solution $S_{1,2}(L)$ parameterized by the variable $K$. Let us illustrate our results with some concrete examples. If $J_{1}(L)= \pm 1$ [or $J_{2}(L)= \pm 1$ ], the line of polarization attraction draws an eight-shaped figure on the Poincaré sphere [see Fig. 2(a)]. In the particular case $\phi=$ $\pi / 4$ and $J_{2}(L)=\varepsilon$, with $\varepsilon= \pm 1$, a straightforward computation leads to the parameterized equation

$$
\left\{\begin{array}{l}
S_{1}= \pm|K| \sqrt{1-K^{2}} \\
S_{2}=-\varepsilon\left(1-K^{2}\right) \\
S_{3}=K
\end{array},\right.
$$

where the first equation implies that $|K| \leq 1$. The two different signs for $S_{1}$ draw the corresponding two parts of the eight-shaped figure. For an arbitrary SOP of the injected pump, the eight curve exhibits a complex deformation, as illustrated in Fig. 2(b). Note that the numerical simulations show that only half of the closed curve computed from our theory can be reached by the spatiotemporal dynamics (see Fig. 2). Indeed, the stationary solutions that lie on the other half-curve exhibit an oscillatory behavior and are unstable [10]. It is also important 

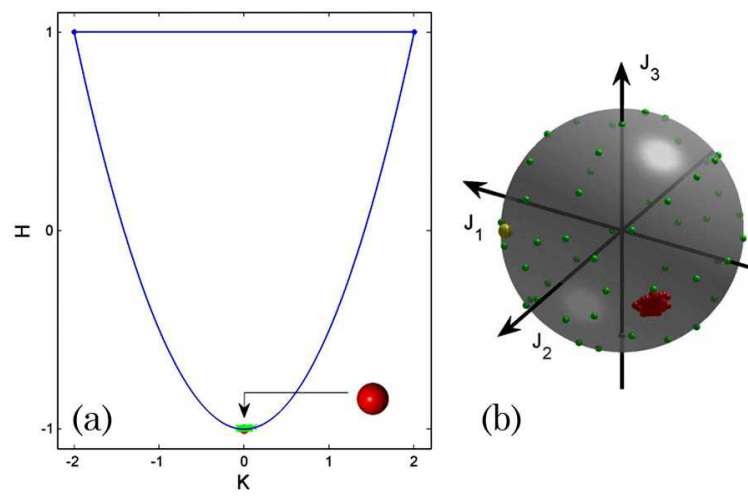

(b)

Fig. 3. (Color online) Same as Figs. 1 and 2 but for the RBF. The polarization attraction is due to the presence of a sphere of singular points (a). All signal SOPs are attracted (up to a sign change) toward the pump SOP $\left[S_{1}(L)=-J_{1}(L), S_{2}(L)=J_{2}(L)\right.$, $S_{3}(L)=-J_{3}(L)$ ], as confirmed by the numerical simulations (b) with $[L=20, \vec{J}(L)=(0.7,0.7,0)]$.

to remark that, as previously pointed out in [7], the polarization attraction becomes less efficient as $J_{3}(L)$ approaches \pm 1 . More precisely, it can be shown that the system relaxes toward values of $K$ belonging to an interval whose length decreases to 0 as $J_{3}(L)$ goes to \pm 1 . In the limit $J_{3}(L)= \pm 1$ (i.e., $J_{1}(L)=J_{2}(L)=0$ ), Eq. (2) no longer depends on $S_{1,2}$ and $H$ is a function of only $\bar{K}$ and $\phi$. This expression of $H$ is not compatible with Eq. (3). This simply means that the corresponding stationary state cannot belong to the line of singular tori in the diagram $(H, K)$.

In the particular case $\phi=0$, the line of bitori in the diagram $(H, K)$ reduces to a single point of coordinates $(H=-1, K=0)$, which belongs to the boundary of the diagram [see Fig. 1(b)]. According to our previous discussion, this qualitative change should lead to a polarization attraction toward a unique SOP on the Poincaré sphere. This is confirmed by the theory and the numerical simulations of Eq. (1). They show that an arbitrary polarization state of the signal is attracted (at $z=L$ ) toward a specific SOP, which is determined by the injected pump SOP, i.e., $S_{3}(L)=-J_{3}(L), S_{1}(L)=J_{1}(L)$ and $S_{2}(L)=$ $-J_{2}(L)$. From a geometrical point of view, note that the bitorus is transformed into a sphere when $\phi=0$ (see Fig. 1).

Polarization attraction in RBFs. It is interesting to note that RBFs exhibit a diagram $(H, K)$ very similar to that of HBSFs with $\phi=0$. We thus study RBFs by considering the model recently derived in [9], in which the counterpropagating interaction is ruled by Eq. (1) with $\mathcal{I}=\operatorname{Diag}(0,0,0)$ and $\mathcal{J}=\operatorname{Diag}(-1,1,-1)$. The stationary system is Hamiltonian with $H=S_{1} J_{1}-S_{2} J_{2}+S_{3} J_{3}$. In this expression of $H$, the three axes play a symmetric role, a property that leads to three constants of motion, $K_{1}=S_{1}+J_{1}, K_{2}=S_{2}-J_{2}$, and $K_{3}=S_{3}+J_{3}$. As for
HBFs with $\phi=0$, the diagram $(H, K)$ for RBFs exhibits a unique singular point associated to a sphere belonging to the boundary of the diagram [see Fig. 3(a)]. Any point

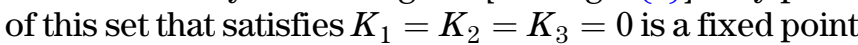
with respect to the stationary Eq. (1). Numerical computations show that this set plays the role of an attractor for the spatiotemporal dynamics. Accordingly, the signal beam is attracted (up to a sign change) by the SOP of the pump, i.e., $S_{1}(L)=-J_{1}(L), S_{2}(L)=J_{2}(L)$, and $S_{3}(L)=-J_{3}(L)$. This remarkable property has been confirmed by the space-time numerical simulations [see Fig. 3(b)].

To conclude, note that, under rather general conditions, for both HBSFs and RBFs the efficiency of the attraction process increases as the fiber length and the powers of the beams increase. We underline that all the above analysis can be extended to unequal signalpump powers: For HBSFs polarization attraction still occurs along a line of polarization states on the Poincaré sphere, while for RBFs the singular torus is shown to split into two distinct singular tori for $S_{0} \neq J_{0}$, whose SOP coordinates read $S_{1}=-\rho J_{1}, S_{2}=\rho J_{2}, S_{3}=-\rho J_{3}$, with $\rho=S_{0} / J_{0}$. For $\rho \simeq 1$ (within 10\%), the simulations reveal an attraction toward the two SOPs states, while for higher values of $\rho$ the system exhibits a complex dynamics, including periodic behaviors that will be the subject of future investigations.

\section{References}

1. E. Heebner, R. S. Bennink, R. W. Boyd, and R. A. Fisher, Opt. Lett. 25, 257 (2000).

2. S. Pitois, A. Picozzi, G. Millot, H. R. Jauslin, and M. Haelterman, Europhys. Lett. 70, 88 (2005).

3. A. Picozzi, Opt. Express 16, 17171 (2008).

4. S. Pitois, G. Millot, and S. Wabnitz, Phys. Rev. Lett. 81, 1409 (1998).

5. S. Pitois, J. Fatome, and G. Millot, Opt. Express 16, 6646 (2008).

6. E. Assémat, S. Lagrange, A. Picozzi, H. R. Jauslin, and D. Sugny, Opt. Lett. 35, 2025 (2010).

7. V. V. Kozlov and S. Wabnitz, Opt. Lett. 35, 3949 (2010).

8. J. Fatome, S. Pitois, P. Morin, and G. Millot, Opt. Express 18, 15311 (2010).

9. V. V. Kozlov, J. Nuno, and S. Wabnitz, J. Opt. Soc. Am. B 28, 100 (2011).

10. D. Sugny, A. Picozzi, S. Lagrange, and H. R. Jauslin, Phys. Rev. Lett. 103, 034102 (2009).

11. S. Lagrange, D. Sugny, A. Picozzi, and H. R. Jauslin, Phys. Rev. E 81, 016202 (2010).

12. R. H. Cushman and L. Bates, Global Aspects of Classical Integrable Systems (Birkhauser, 1997).

13. D. Sugny, P. Mardesic, M. Pelletier, J. Jebrane, and H. R. Jauslin, J. Math. Phys. 49, 042701 (2008).

14. E. Assémat, A. Picozzi, H. R. Jauslin, and D. Sugny, Optical Polarization Control: A Pedagogical Approach to New Hamiltonian Tools, in preparation (2011). 